\title{
Promising a Safe Futuristic Hope to Covid-19 Pandemic through Vaccination - A Review of Different Vaccines
}

\section{Manisha Saxena $^{1 *}$, Neel Kant Patil ${ }^{2}$, Mohit Sareen ${ }^{3}$, Manoj Meena ${ }^{3}$ and Nitesh Tyagi ${ }^{4}$}

${ }^{1} P G$ Student, Rajasthan Dental College and Hospital, Jaipur, India

${ }^{2}$ Professor and HOD, Rajasthan Dental College and Hospital, Jaipur, India

${ }^{3}$ Reader, Rajasthan Dental College and Hospital, Jaipur, India

${ }^{4}$ Lecturer, Rajasthan Dental College and Hospital, Jaipur, India

*Corresponding Author: Manisha Saxena, PG Student, Rajasthan Dental College

and Hospital, Jaipur, India.
Received: June 22, 2021

Published: July 28, 2021

(C) All rights are reserved by Manisha Saxena., et al.

\begin{abstract}
The current pandemic of coronavirus disease (COVID-19) has influenced the world socially and economically. COVID-19 is originated due to a new strain of coronavirus known as severe acute respiratory syndrome coronavirus-2 (SARS-CoV-2). The world is trying to control epidemic by depending on social distancing and hygiene-related measures, along with trial of different drug combination. The world is trying to put measures collectively to return to pre-pandemic condition, and an initiative is put forwarded in development of vaccine as protection against SARS-CoV-2. Till date, thirteen vaccines have been approved for application and more than 90 vaccine candidates are under clinical trials. This review focuses on the summary of development of vaccine in relation to COVID-19. A brief introduction of COVID-19 variants is also mentioned. The development of a vaccine has already started, and headways are still going. However, the world population will have to remodel to the "new normal" and implement social distancing and hygienic measures, along with the vaccines till the effect of pandemic goes off.
\end{abstract}

Keywords: Vaccine; COVID-19; Global Pandemic; Coronavirus; SARS-CoV-2

The COVID-19 pandemic, also known as the coronavirus pandemic, is an ongoing global pandemic of coronavirus disease 2019 (COVID-19), which is caused by severe acute respiratory syndrome coronavirus 2 (SARS-CoV-2). The virus was first pin down in December 2019 in Wuhan, China. The World Health Organization declared a Public Health Emergency of International Concern (PHEIC) regarding COVID-19 on 30 January 2020, and later declared a pandemic on 11 March 2020 [1]. As of 10 June 2021, more than 174 million cases have been confirmed, with more than 3.75 mil- lion confirmed deaths attributed to COVID-19, making it one of the deadliest pandemics in history [2].

Human coronaviruses are responsible for causing disease that are categorized from the common cold to more severe diseases such as Middle East respiratory syndrome (MERS, fatality rate $\sim 34 \%$ ). SARS-CoV-2 ranking is seventh in coronavirus family to infect people, after 229E, NL63, OC43, HKU1, MERS-CoV, and the original SARS-CoV [1]. 
Severe acute respiratory syndrome coronavirus 2 (SARS-CoV-2) is the culprit virus behind COVID-19.

The virus can be transmitted from asymptomatic or symptomatic patients. As no specific treatment is available, the control of disease is challenging task. An effective approved vaccine is necessary to control the pandemic. Quarantine is effective but it cannot be the only measure to prevent the spread as it has negative impact on economy of the countries. Therefore, an effective and safe vaccine against COVID-19 is the need of an hour [3].

Vaccines is preventive medication in public health to control and limit the spread of infectious diseases. The use of vaccinations has proved boon to control and eradicate many diseases in the past [1].

The availability of vaccines, combined with the operational expertise and political commitment to administer them, is responsible for many of the most important achievements in public health in human history. A vaccine vanquished smallpox, the only human disease that has been completely eradicated from the world. Polio, a menace for children and parents for a long time, is also on the border of elimination because of vaccines. The spread of measles, another disease that primarily affects children has also been brought down to low level by vaccination drive.

Edward Jenner's initiative to develop a smallpox vaccine marks the modern era of vaccine. The "Golden Age" of vaccine development is recent, related with the advent of virus propagation in cell culture in early 1950s. This technological breakthrough made vaccines such as Jonas Salk's polio vaccine possible and has led to the diversity of vaccine development platforms today [4].

\section{Development of vaccine}

The development of vaccines offers a unique set of challenges as compared to other drugs. Vaccines are usually administered to healthy people who may never have contracted the disease against which the vaccine offers protection. Therefore, vaccines have a higher bar for safety and tolerability than other drugs. While recipients might be willing to experience some discomfort at the injection site, severe reactions that occur with any sort of regularity will inhibit the use of the vaccine.

Vaccine modalities include live, attenuated viruses, inactivated pathogens, protein subunit vaccines, polysaccharides, conjugate vaccines, DNA platforms, mRNA platforms, and viral vectors. The choice of platform for the vaccine plays a crucial role in both the elicited immune response and the duration of protection provided by the final product [4].

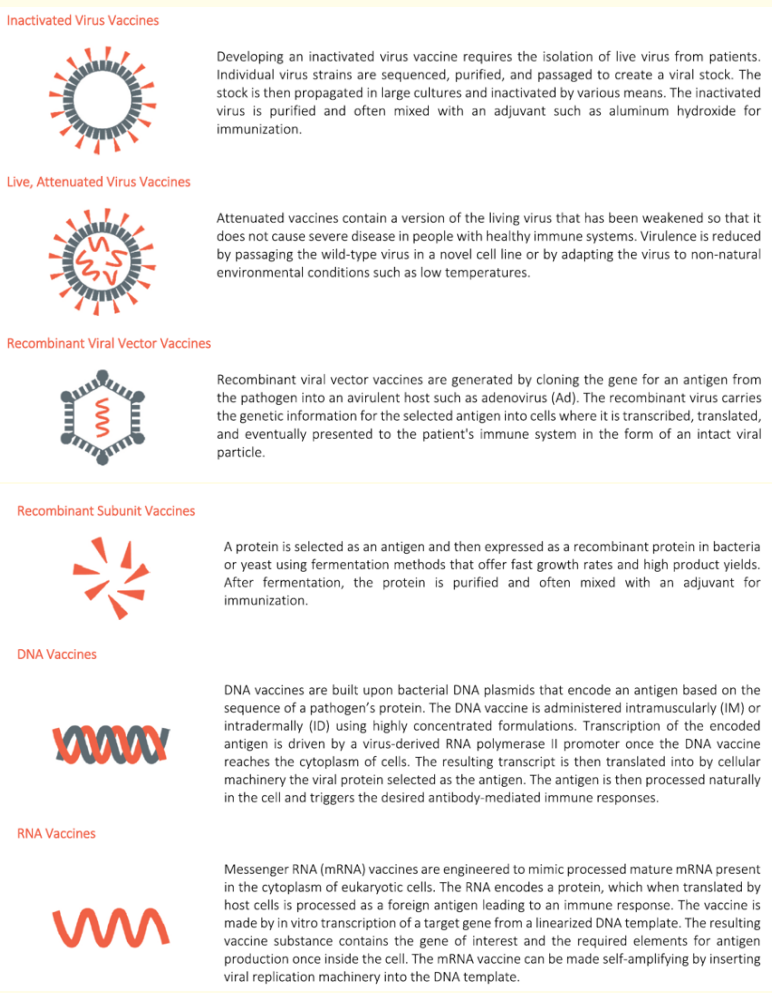

Figure 1: Different types of vaccines [4].

The major epidemics declared by the WHO such as the arboviral diseases Dengue, Chikungunya and Zika, which reached trials in 52 years, 19 years and 9 years after declaration of major outbreaks, respectively. The MERS-CoV and SARS-CoV have progressed to clinical trials within 22 months and 25 months, respectively after their outbreaks, whereas the mRNA1273-COVID-19 vaccine was ready for trials (NCT04283461) in only 69 days after the identification of the SARS-CoV-2 as the causative agent of the current outbreak. This pace of development is amazing and can be attributed to advancement of vaccine development [1].

\section{Principles of vaccine}

The genome sequences of SARS-CoV-2 and SARS-CoV are highly similar, and both viruses have ACE2 as cellular receptor. This 
knowledge served as the basis to outline the development of SARSCoV-2 vaccines. The SARS-CoV-2 has gene sequence which is $89 \%$ identical to bat SARS-like coronavirus. The nucleotide sequence has shown $96.2 \%$ similarity with the one of bat coronavirus at the whole genome level, the primary reservoir of SARS-CoV-2 has not yet been determined [5].

The "new normal" approach of social distancing, wearing a mask, and screening of temperature by infrared thermometer, are preventive measures. Proper scientific approach through vaccine and accurate drug treatment is important to for taking life on proper track as it was before pandemic. The countries across the world are trying hard to find a cure for this virus [5].

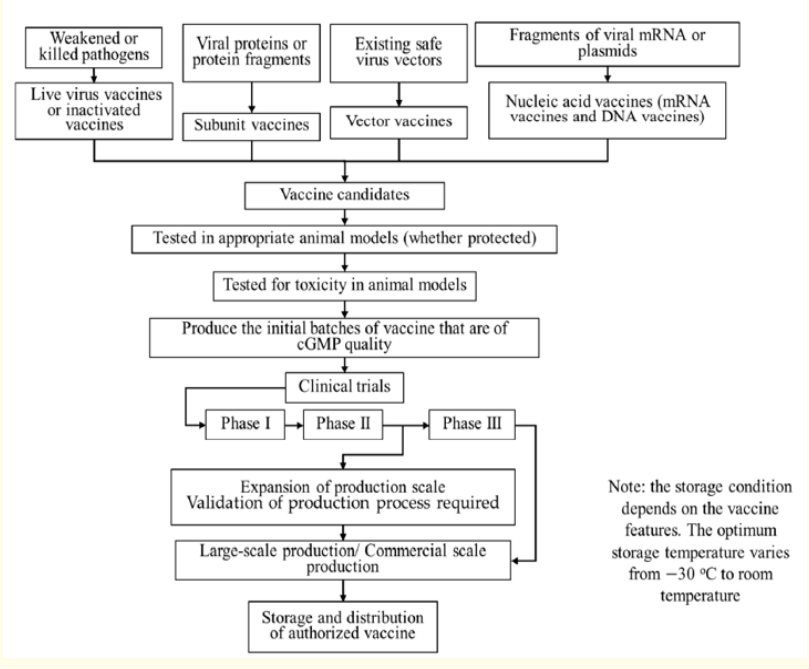

Figure 2: Flow chart of vaccine development from initial stages to clinical trials and supply using logistic chain for vaccine distribution [5].

Till now, there are more than 90 vaccine candidates are in clinical development process, undergoing in different Phases of clinical trials. WHO has standardized the success rate for COVID-19 vaccines providing highest protection in one year with $70 \%$ efficacy and the lowest protection for 6 months with 50\% efficacy. The developed vaccines have to be safe and effective for the public. The vaccines will have to satisfy all regulatory parameters in quality, efficacy, and safety terms before a marketing authorisation is granted [5].
Previously, the monitoring of vaccine safety after licensure is guided by passive and active surveillance. The passive surveillance system is the basis of pharmacovigilance, where data from recent reports of suspected adverse drug reactions (ADRs) and adverse events following immunisation (AEFI) from physicians and patients are collected. Eudra Vigilance (EV) is a passive pharmacovigilance system which collects, manage and analyse suspected ADRs and AEFI reports for medicines approved in the EU and it is operated by the EMA (European Medical Agency).

The monitoring of safety reports of drugs and vaccines is important as clinical trials assess primarily the efficacy; safety is kept as a secondary objective. The trials are able to recognize only common adverse events such as local and systemic reactions related to the immunogenicity of the vaccine that are noticed soon after administration. The trials are unable to notice rare adverse events that occur with trials occur in larger populations or as a delayed reactions occurring after a long-term use; therefore, pharmacovigilance and post marketing surveillance is of considerable significance to detect unusual adverse events [8].

Severe acute respiratory syndrome coronavirus 2 (SARS-CoV-2) is a causative agent for the respiratory ailment known as coronavirus disease 2019 (COVID-19). SARS-CoV-2 is an envelope virus 50 - 200 nanometers in diameter. It expresses several genes required for transmission and propagation, including those encoding spike (S) proteins that allow the virus to attach to and fuse with the infected host's cells. The RNA genome is organized and maintained within the envelope [4].

\section{Covid-19 biology and current COVID-19 vaccines}

Coronaviruses comes under the Coronaviridae family. It is the largest group of host-specific RNA viruses that can infect humans, birds, snakes, bats, and mammals.

Coronavirus name is owning to crown-like surface projections or the Sun's corona. It is non-segmented, enveloped, single-strand, positive-sense, ribonucleic acid viruses.

The SARS-CoV-2 virus consists of four basic proteins related to structure:

1. S-spike protein (outer spiky glycoprotein),

2. Envelope protein (E), 


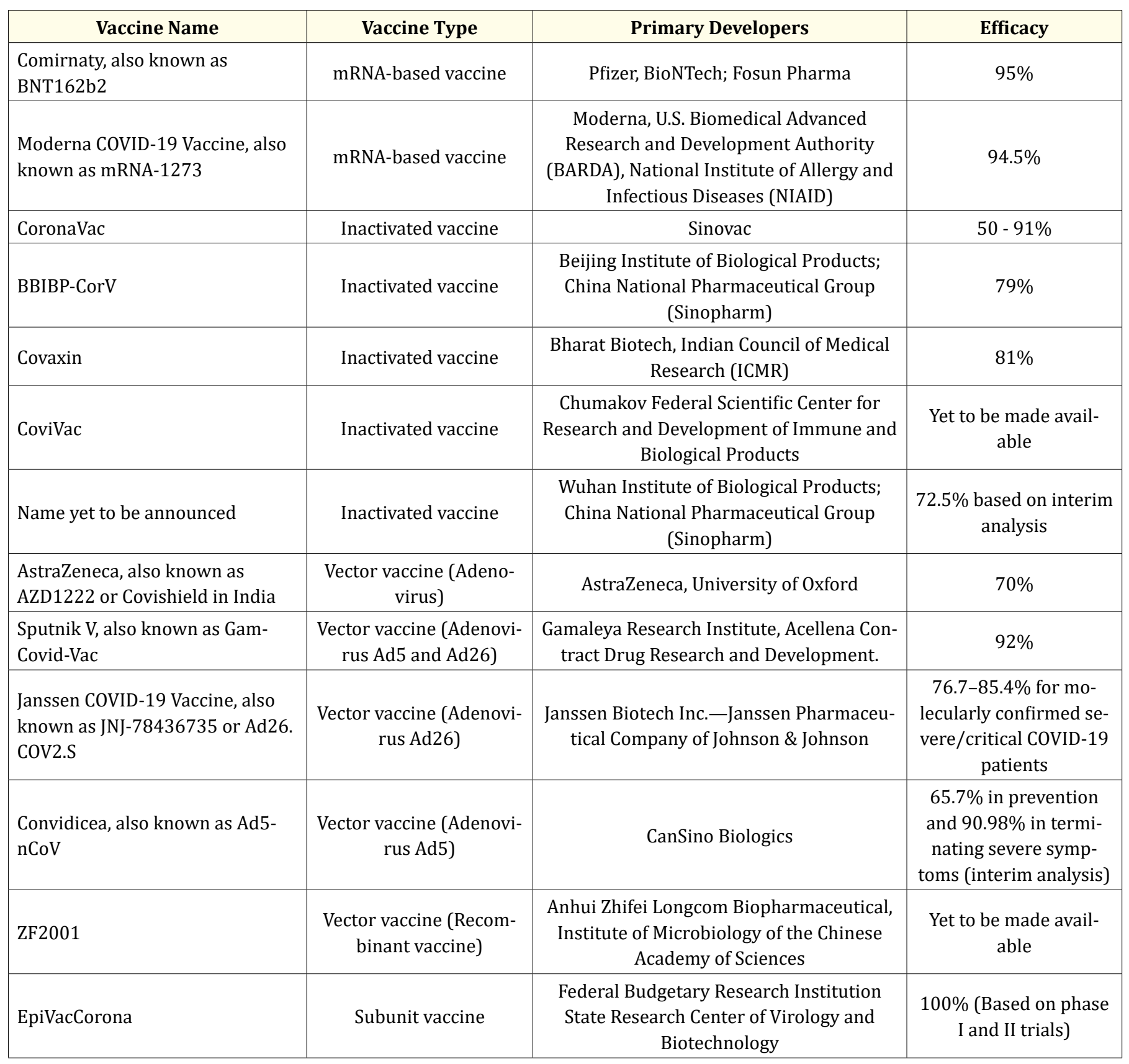

Table 1: Different approved vaccines available for COVID- 19 are arranged according to vaccine types in March 2021 [5].

3. Membrane glycoprotein (M),

4. $\quad$ Nucleocapsid protein $(\mathrm{N})$,

That can hamper with the host's immune system, enhancing the attachment and transportation into host cells. The S protein consists of two domains-the S1 domain that contains the receptorbinding domain (RBD) mediating the attachment to the receptor cell, and the S2 domain, that aids the virus fusion to the host cell.

The entry of SARS Cov 2 into host cell is mediated by binding of the receptor binding domain of $\mathrm{S}$ protein with ACE 2 cells of the 


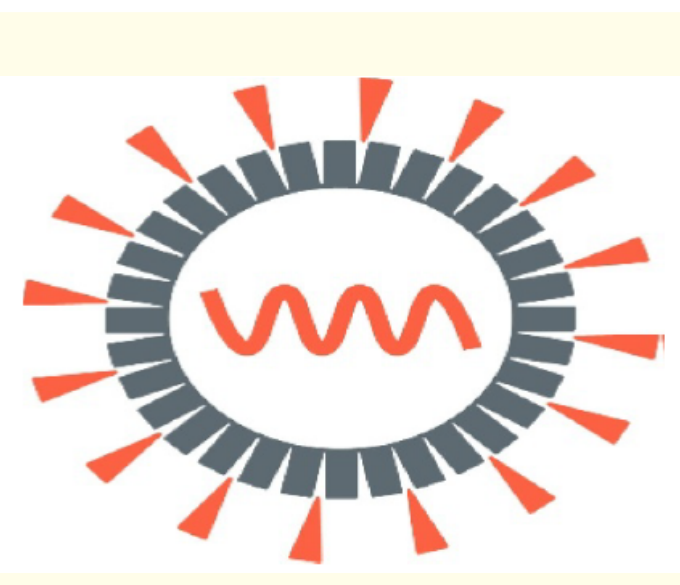

Figure 3: Structure of SARS-CoV-2.

host. S protein is the primary target of vaccine development [6]. The mechanical stability of SARS-CoV-2 RBD (250 pN) COVID-19 due to stronger intermolecular interactions as compared to RBD of SARS-COV ( $200 \mathrm{pN}$ ) and is responsible for further spread. The M and E proteins are accountable for virus assembly, and the N protein plays a vital role in RNA synthesis [5].

The presently available COVID 19 vaccine uses various platforms.

\section{Traditional inactivated whole-virus vaccines}

These vaccines depend on living virus that has been killed or inactivated, not causing the clinical manifestation of disease. This technology is being used for long time for vaccine preparation. The idle viruses keep their replicative property in vivo without causing major symptoms.

The viruses boost the immune system and prompt a robust and long-lasting immune response that prevents infection. Inactivated whole virus includes the entire disease-causing virion (a complete, infective form of a virus outside a host cell, with a core of RNA and a capsid), which is made inert physically by heat or chemically. The emerging immune response is responsible for providing protection against many other SARS-CoV-2 antigens. These vaccines have several benefits, such as low priced, safety, absence of genetic manipulation. The Sinopharm's Wuhan Institute of biological products and the Chinese company Sinovac Biotech produce such types of vaccines [6]. An inactivated vaccine developed by Beijing Biological
Products Institute under Sinopharm's subsidiary, China National Biotec Group (CNBG), was China's first COVID-19 vaccine to receive conditional approval for the country's domestic market on December 31, 2020. It was followed by CoronaVac, developed by Chinese pharmaceutical firm Sinovac Biotech [7].

Live virus vaccine

Live virus vaccine is manufactured by the virus with diminished virulence or even non-virulence. Codagenix and the Serum Institute of India are developing a live serum attenuated vaccine based on the CodaVax technology under the Phase I trial.

\section{Subunit vaccines}

Subunit vaccines are composed of non-genetic viral proteins or peptide fragments to initiate strong immune responses. The absence of an entire infectious virus in subunit vaccines is responsible for an increase in the safety parameters and eliminates the problem of virus inactivation or reversal of toxicity. Research shows that most of the SARS-CoV-2 subunit vaccines target proteins, particularly the spike protein, or age proteins in particular areas; while some other subunit vaccines are directed on $\mathrm{N}$ proteins. Its purpose is to deliver antibodies and to contain human lymphocyte antigen (HLA) restricted T cell epitopes. Israeli company MigVax is taking keen interest on developing an oral anti-COVID-19 subunit vaccine based on earlier research product [5].

\section{mRNA-based vaccines}

In the RNA (Ribonucleic Acid) vaccines or mRNA (messenger RNA) vaccines transient artificially created molecules of the RNA sequence transfected (infected by transformation) by COVID-19 virus are administer in the individual. The infected vaccine's RNA behaves as mRNA inside the immune cells of the vaccinated individual and initiate the cells to produce a foreign protein that would usually be produced by a virus. mRNA is transported into the human cell in multiple ways, commonly by lipid microvesicles (liposomes). The mRNA vaccine is much easier to create and design, quick to produce, and stimulates cellular immunity, as well as humoral immunity.

The lipid nanoparticles (LNPs) are the customarily used vectors for in vivo administration of mRNA vaccine. Indeed, LNPs guard the mRNA against degradation, can be co-delivered with adjuvants, can 
be manufactured with relative ease, can be targeted to the desired cell type by surface decoration with ligands, and expedite endosomal escape.

These vaccines are to be kept at -30-to--80-degree C.

The Pfizer-BioNTech and Moderna COVID-19 mRNA vaccines belong to this category.

\section{Adenoviral vector vaccines}

In adenoviral vector vaccines, a DNA gene specific to the virus being focused is added to the viral vector. These kinds of viral vectors are viruses from chimpanzees, gorillas or human adenoviruses. A pre-existing immunity against the virus vector may affect vaccine efficacy. This gene shunt into a human cell using viral vectors. This gene ciphers the S protein, which is only found on the surface of SARS-CoV-2. The viral vector uses this gene when it reaches inside the cell and produce the $S$ protein and show it on the cell's surface. The virus in which the DNA is inserted may drop its ability to replicate. It is usually given by intramuscular route.

Non-replicating vectors are used in Astra Zeneca, Sputnik V Gam-COVID-Vaccine and Johnson \& Johnson [6].

Vaccines in India

Covishield

The Oxford-AstraZeneca vaccine is produced locally by the Serum Institute of India, the world's largest vaccine manufacturer. The vaccine is made from a diminished variety of a common cold virus (known as an adenovirus) from chimpanzees. It has been redesigned to look more like coronavirus - although it can't cause ailment.

When the vaccine is injected into a patient, it triggers the immune system for making antibodies and prepares it to attack any coronavirus infection.

The jab is delivered in two doses given between the gap of four and twelve weeks. It can be safely stored at temperatures of $2 \mathrm{C}$ to 8C.

The jab developed by Pfizer-BioNTech, which is currently dispensed in several countries, must be stored at -70C and can only be shifted for a limited number of times - a massive challenge in India, where temperatures reach $50 \mathrm{C}$ in summer.
Studies conducted on Oxford-AstraZeneca vaccine have shown promising result that when people were given a half dose and then a full dose, effectiveness hit $90 \%$.

Covaxin

The constituent of COVAXIN is killed corona virus, that means it is inactivated vaccine making it safe to be administered into the body.

National Institute of Virology (NIV) in collaboration with Indian council of Medical Research (ICMR) isolated the corona virus sample and inactivated vaccine is developed and manufactured in Bharat Biotech's BSL-3 (Bio-Safety Level 3) high containment facility. It is the India's first vaccine against Corona Virus which has been completely developed by India. The vaccine is made using WholeVirion Inactivated Vero Cell derived platform technology.

When administered, it acts as immune potentiator that boost and increase the immune response of an individual. The body recognizes the passive virus cells, prompting the immune system to make antibodies against the virus. It is two dose vaccination regimes given with a gap of four weeks. It is a vaccine with no sub-zero storage, no reconstitution requirement, and ready to use liquid presentation in multi-dose vials, stable at $2-8^{\circ} \mathrm{C}$. The preliminary data from its Phase 3 clinical trial showed its efficacy rate as $81 \%$.

Drug controller of India (DGCI) gave an emergency use approval in January while the third phase of the trial was still underway, and crucial to prove its efficacy.

\section{Sputnik V}

Sputnik V also known as Gam-COVID-Vac, the vaccine uses a heterologous recombinant adenovirus approach using adenovirus 26 (Ad26) and adenovirus 5 (Ad5) as vectors for the expression of the severe acute respiratory syndrome coronavirus 2 (SARS-CoV-2) spike protein. The use of two varying serotypes, which are given 21 days apart, is considered to overcome any pre-existing adenovirus immunity in the population. This is the only vaccine which is using two different types of vectors. The idea behind using two different vector formulas is that it boosts the immune system even more than using the same version twice - and may give longer-standing protection.

The vaccine, developed by Moscow's Gamaleya Institute. 
The vaccine-infected cells are eventually destroyed by the very body's immunity. They are unable to replicate, but trigger the innate immunity sensors sufficiently to ensure robust immune system. The individual is exposed to virus genetic code allows it to identify the threat and prepare antibody, without risking the life. It is easier to transport and store at temperatures of between 2 and 8C degrees.

Hyderabad-based company Dr Reddy's Laboratories has imported the first batch of 150000 doses to India [9].

\section{ZyCov-D}

ZyCov-D, the Zydus' Covid-19 vaccine, is being developed by Ahmedabad-based Zydus-Cadila with support from the Centre's National Biopharma Mission as part of Biotechnology Industry Research Assistance Council, department of biotechnology. Zydus Cadila is testing its Covid 19 vaccine candidate on children in the age group of 12 - 18 years, apart from adults [13]. ZyCoV-D, the DNA vaccine against Covid-19 developed on its way to becoming the first approved DNA Covid vaccine in the world. ZyCoV-D does so with the help of a plasmid - a DNA fragment capable of surviving outside the nucleus of a cell. Both ZyCoV-D, which uses this technology, and the vaccines developed by American companies Pfizer and Moderna come under what is known as "nucleic acid vaccines". The only difference is that the latter two use a messenger RNA to essentially carry the same message into the body [14].

\section{Other vaccine candidates}

The other candidates which are in different stages of trials in India to test safety and efficacy include:

- A vaccine being developed by Hyderabad-based Biological E, the first Indian private vaccine-making company. Biological E's vaccine - to be called Corbevax -- is similar to the Novavax vaccine, which is more than 90 per cent effective including against Covid variants, according to the company. Novavax will be produced in India by the Serum Institute of India, which also makes Oxford-AstraZeneca's Covishield [15].

- $\quad$ The mRNA vaccine candidate - HGC019 - has been developed by Pune-based company Gennova and is supported with a seed grant under the Ind-CEPI mission of the Department of Biotechnology under the Ministry of Science and Technology [16].
- A nasal vaccine BBV154 by Bharat BioTech.

- A second vaccine being developed by Serum Institute of India and American vaccine development company Novavax [9].

\section{Efficacy and safety}

Till now many vaccines have been authorised for use in many countries, which differs in terms of efficacy against COVID-19. The efficacy of the vaccine Comirnaty (BNT162b2) of Pfizer is 95\%, succeeded by Moderna COVID-19 Vaccine (mRNA-1273) of 94.5\%. The vaccine BBIBP-CorV showed 86\% efficacy, while BNT162b2 $(87 \%)$ is less effective in the prevention of severe disease. The foundation of efficacious and safe vaccines is urgently required for pandemic control [5].

\section{General therapeutic considerations}

The first COVID-19 vaccine was given regulatory approval on 2 December by the UK medicines regulator MHRA. It was evaluated for emergency use authorization (EUA) status by the US FDA, and in several other countries. A COVID-19 vaccine is a vaccine directed to provide acquired immunity against severe acute respiratory syndrome coronavirus 2 (SARS-CoV-2), the virus that causes coronavirus disease 2019. (COVID-19) On 10 January 2020, the SARS-CoV-2 genetic sequence data was shared through GISAID, and by 19 March, the global pharmaceutical industry declared a major development to address COVID-19. The Phase III trials of several COVID-19 vaccines have shown efficacy as high as $95 \%$ in preventing symptoms of COVID-19 infections. As of June 2021, 18 vaccines are authorized by at least one national regulatory authority for public use that include two mRNA vaccines, nine inactivated virus vaccines, five viral vector vaccines and two protein sub-unit vaccines [12].

The polyethylene glycol (PEG) excipient, also known as macrogol, has been used for the first time in Pfizer and moderna COVID-19 vaccines.

The motive behind using it is to stabilize lipid nanoparticles that contain the mRNA. The PEG used in these vaccines differ from PEG used in other type of products in terms of molecular weight and using them as stabilising component of vaccine. The lipid nanoparticle (LNP) carrier system to use to prevent rapid enzymatic break- 
down of mRNA and ease in vivo delivery. The added PEG further stabilizes the lipid-based nanoparticle carrier system by providing hydrophilic layer for increasing the half-life.

As compared to PEG, excipient polysorbate 80 , also known as

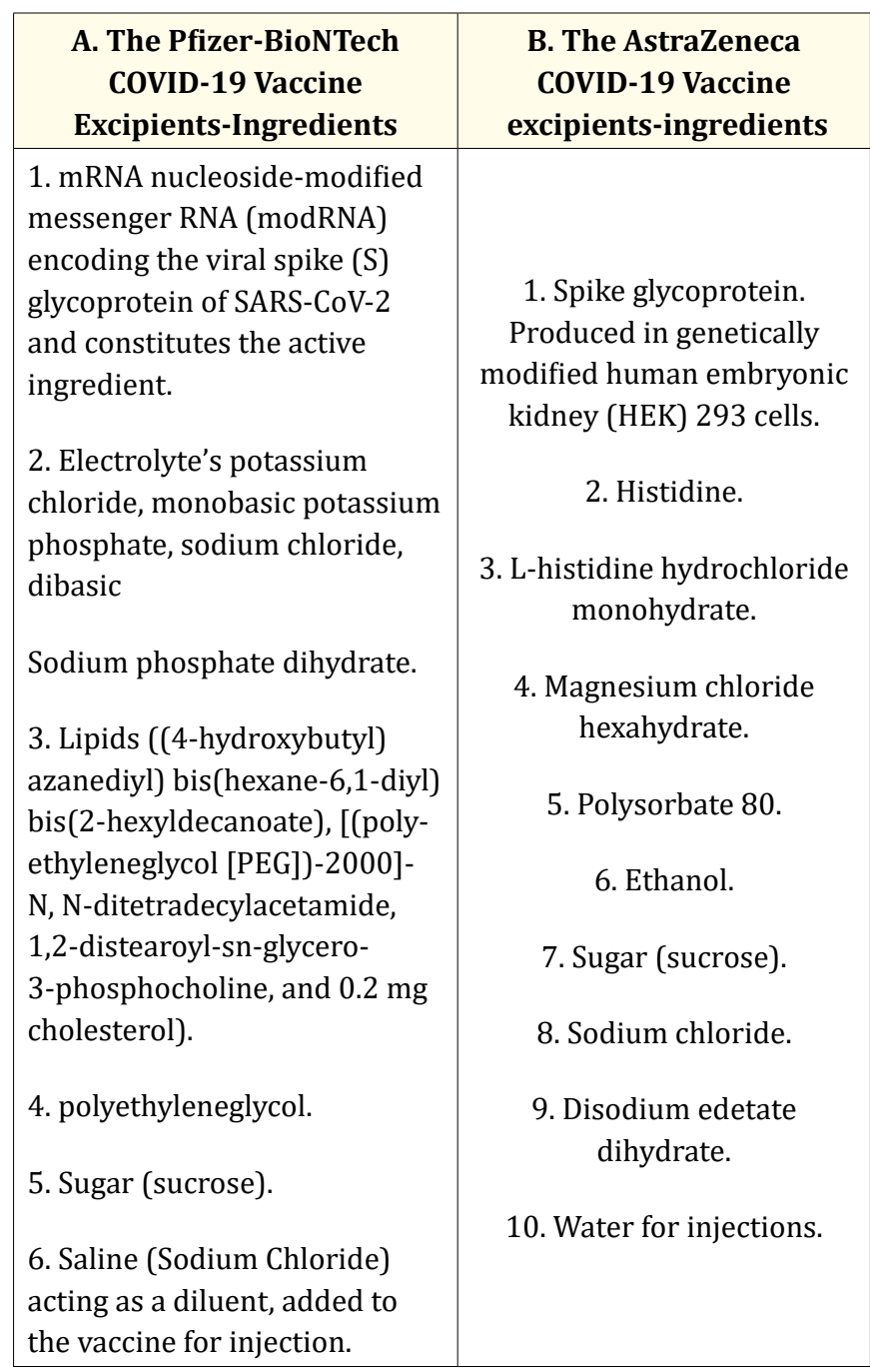

Table 2: Ingredients of Pfizer and AstraZeneca COVID-19 vaccines [6].

Tween 80, has been used in AstraZeneca vaccine and Johnson and Johnson vaccine.

The ingredients of 2 main vaccines are listed in table 2 .

The individual's past medical history should be checked for any previous severe or mild allergic reactions to any vaccine's constituent beforehand. The Centres for Disease Control (CDC) and the
World Allergy Organization Anaphylaxis Committee have instructed safety guidelines concerning vaccination of an individual with history of allergic reactions. Individuals with common allergies to food, medications, and insect bites need to be more alert and careful while taking vaccines.

Anyone with a history of immediate allergic reaction of any severity to any component of mRNA COVID-19 vaccines or to polyethylene glycol or polysorbate should not receive Pfizer-BioNTech or Moderna COVID-19 vaccine. Individuals with a latex allergy should be vaccinated in latex free surroundings.

An allergists advice and directions are of great importance. An anaphylactic reaction is a severe allergic reaction leading to life threatening conditions with sudden onset within minutes. The arousal of anaphylactic reactions may be delayed, and cardiovascular collapse and/or respiratory failure can occur up to eight hours

The decalogue of treatment measures should be applied in the following order.

1. Patient in recline position with legs up and administer intramuscular epinephrine

2. Intravenous line for volume replacement with intravenous $0.9 \% \mathrm{NaCl}$

3. Airways clearing, vital signs checking, oxygen via facial mask at least $10 \mathrm{~L} /$ minute should be administered.

4. 2 - 3L of intravenous $0.9 \% \mathrm{NaCl}$ in $10-20$ min if hypotension and rapid volume loss occurs.

5. Repeat intramuscular epinephrine if no improvement within 5 - $10 \mathrm{~min}$ and call emergency assistance

6. Short-acting beta-agonists [salbutamol] puffs via large volume spacer for severe dyspnea/wheezing

7. Glucagon in patients on beta-blockers who are unresponsive to epinephrine.

8. Nebulized epinephrine and nebulized short-acting betaagonists in cases of signs of severe upper airway obstruction. (laryngeal/uvula/tongue edema).

9. Oral or intravenous glucocorticoids and oral or intravenous antihistamines are given.

10. Measuring mast cell tryptase 2 - $3 \mathrm{~h}$ after the beginning of the reaction to confirm anaphylaxis.

Table 3: Measures to be taken when anaphylaxis occurs after giving vaccine [6]. 
after symptom initiation. Therefore, the initial and usually milder symptoms should also be monitored from the beginning. The Pfizer/BioNTech vaccine, has shown 11.1 cases of allergic reactions (including anaphylaxis) per one million doses, and precisely, 71\% of reactions occurred within 15 min following the first dose. Epinephrine is the drug of choice in the treatment of anaphylaxis [6].

\section{Corona virus variants}

A virus variant is an isolate whose genome sequence differs from that of a reference virus. All viruses including Sars Cov-2, change over time. Genetic variants of SARS-CoV-2 have been developing due to viral mutation.

\section{The classification of SARS-CoV-2 variant}

Variant classification scheme that defines three classes of SARSCoV-2 variants is put forward by US government interagency group developed:

1. Variant of interest

2. Variant of concern - The B.1.1.7, B.1.351, P.1, B.1.427, and B.1.429 variants circulating in the United States are classified as variants of concern.

3. Variant of high consequence__no variants of high consequence have been identified in the United States [10].

Presently five variants are observed in US:

- B.1.1.7: It was initially isolated in the United Kingdom but identified firstly in the United States in December 2020.

- B.1.351: It was initially detected in South Africa in December 2020, but noticed in US in January 2021.

- P.1: P.1 was initially identified in travellers from Brazil.

- $\quad$ B.1.427 and B.1.429: These two variants were first noticed in California in February 2021 [9].

\section{Inception of variants}

Variants develop when original virus develops mutation. Mutations are necessary to make them grow and survive in new host. Most of the mutations are deleterious for the virus, but some make it easier for the virus to survive. The SARS-CoV-2 virus is evolving at rapid rate as it is infecting large number of people. High levels of circulation make the virus multiply at faster rate.

The B.1.617 variant of the virus has two mutations, known as
E484Q and L452R.These two mutations are reported together for the first time in India although they have been seen separately in other corona viruses. WHO also classified it as Variant of interest. The L452R mutation is noticed in B.1.427/ B.1.429, which is responsible for increasing their transmissibility. The B.1.617 of the Covid-19 is the fourth variant to be nominated as variant of concern globally. The other three VOC are strains of UK, South Africa and Brazil. It definitely needs more systematic approach for tracking. The B.1.617 variant of the Covid-19, known more commonly as the "double mutant strain" suggests that it involves two variants of the virus. The E484Q mutation is responsible for high transmission ability of Brazilian and South African variants. The L452R mutation, helps the virus to avoid the body's immune retaliation. The double mutation strain was subsequently named B.1.617. The B.1.617.1 and B.1.617.2 variants of the Covid-19, first spotted in India, has been titled as 'Kappa' and 'Delta' respectively by World Health Organisation. (WHO) The name was given according to Greek alphabets that are used to name other corona virus variants. The WHO's gave this name after India objected to call the B.1.617 mutant of the novel coronavirus an "Indian Variant" in media reports [11].

\section{Future of vaccines}

Trials are to be done to get vaccines for children. Different routes of administration need to be developed in order to overcome fear of needle injury. Vaccine escape should be avoided as new mutation could render virus unaffected by vaccines. If vaccines are rendered ineffective against mutated virus, infection rate will boom due to uncontrolled replication of Sars Cov-2 virus. If this condition arrives, then periodic upgradation of vaccine is a must with yearly vaccine immunisation against the coronavirus [5].

\section{Conclusion}

The COVID-19 pandemic has culminated in public health dilemma and economic deterioration, including the largest global recession since the Great Depression of the 1930s.It has increased our focus on vaccines. The consensus is that a return to some kind of "normal" can only occur once effective vaccines are widely available. One of the biggest hurdles for companies is deciding which vaccine platform to pursue to target SARS-CoV-2. There is no precedent for what will work against a novel coronavirus, so nearly every vaccine platform is being explored as COVID-19 vaccines. The rapid development timeline for a COVID-19 vaccine raises doubt in 
everyone mind. On average, the typical vaccine development takes almost a decade. A lot of that time is spent running large clinical trials that consider how a vaccine performs in diverse populations. The 12- to 18-month timeline discussed for a COVID- 19 vaccine comes at the cost of demonstrating safety and effectiveness in every demographic category, regardless of age, race, ethnicity, or gender. The data needed to ensure a vaccine meets everyone's needs, irrespective of their demographic, is challenging to obtain when starting development from scratch, such as with SARS-CoV-2. A vaccine based on a particular platform may work better for certain groups because of its safety profile, delivery method, stability during transport, or it just may be faster to produce and require smaller manufacturing footprints. Regardless of the specific virus or variant, companies will still need to consider how to ramp up their manufacturing to an unprecedented speed while controlling cost, safety, and immunogenicity. They will also need to commit to robust post- marketing pharmacovigilance to identify rare side ef-

\section{Bibliography}

1. Covid-19 pandemic-Wikipedia.

2. World Health Organization. WHO Coronavirus Disease (COVID-19) Dashboard. World Health Organization (2020).

3. Young Chan Kim. "COVID-19 vaccines: breaking record times to first-in-human trials”. npj Vaccines 5 (2020): 34.

4. Mark Verdecia., et al. "COVID-19 vaccine platforms: Delivering on a promise? Human Vaccines And Immunotherapeutics (2021).

5. Yuxin Yan., et al. "The COVID-19 Vaccines: Recent Development, Challenges and Prospects". Vaccines 9 (2021): 349.

6. Nicholas G Kounis., et al. "Allergic Reactions to Current Available COVID-19 Vaccinations: Pathophysiology, Causality, and Therapeutic Considerations". Vaccines 9 (2021): 221.

7. https://www.globaltimes.cn

8. Tobaiqy., et al. "Analysis of Thrombotic Adverse Reactions of COVID-19 Astra Zeneca Vaccine Reported to Eudra Vigilance Database Mansour". Vaccines 9 (2021): 393.

9. www.bbc.com
10. www.cdc.gov

11. www.business-standard.com

12. COVID-19 wikipedia.

13. www.hindustantimes.com

14. https://theprint.in

15. ndtv.com

16. https://www.livemint.com

Volume 5 Issue 8 August 2021

(C) All rights are reserved by Manisha Saxena., et al. 